\title{
FORRAGEAMENTO E MANUTENÇÃO DE COLÔNIAS DO CUPIM Nasutitermes sp (ISOPTERA: TERMITIDAE) EM LABORATÓRIO
}

Rodrigo dos Santos Pinheiro, Vânia Maria Ramos, Renato Marcos de Leão, Viviane Tavares de Almeida, João Vitor Souza Cruz, Matheus Barbosa Saqueti, Matheus Venâncio Prado, Gabriel Ribeiro Milhorança

Universidade do Oeste Paulista - UNOESTE, Curso de Agronomia, Presidente Prudente, SP. E-mail: vaniaramos@unoeste.br

\section{RESUMO}

Os cupins são insetos sociais da ordem Isoptera, com cerca de 2.900 espécies catalogadas no mundo. No Brasil, são várias as espécies de importância agrícola e florestal. Caracterizam-se por colônias complexas do ponto de vista social e estrutural, muitas vezes subterrâneas, o que dificulta o seu completo conhecimento. Diante deste fato, este trabalho teve como objetivo a manutenção de colônias de cupins com importância agrícola em laboratório, do gênero Nasutitermes, para determinar as condições necessárias ao seu desenvolvimento, incluindo preferências de forrageamento por diferentes partes de Paspalum notatum. Colônias jovens foram coletadas em campo para manutenção em laboratório, sob um sistema artificial com condições estruturais (abrigo) e ambientais (luz, temperatura, umidade relativa do ar) que buscaram ser as mais similares possíveis àquelas naturais. Diferentes partes de $P$. notatum foram oferecidos como opção alimentar, em teste com chance de escolha. Após estabelecimento da criação laboratorial e observação do forrageamento de colônias do cupim Nasutitermes sp, verificou-se a preferência por raiz, seguida de fragmentos em decomposição, folhas secas e frescas, equivalentemente. Verificou-se também sua adaptação à um sistema estrutural artificial composto por caixa central e caixa de alimentação, permitindo a manutenção das colônias por tempo mínimo de 5 meses.

Palavras-chave: criação de cupins em laboratório; insetos sociais; pragas de gramíneas; pragas florestais; térmitas.

FORAGING AND MAINTENANCE OF Nasutitermes sp COLONIES (ISOPTERA: Termitidae) IN LABORATORY

\begin{abstract}
Termites are social insects of Isoptera order, with about 2,900 species cataloged in the world. In Brazil, there are several species of agricultural and forestry importance. They are characterized by complex colonies from the social and structural point of view, often subterranean, which hinders their complete knowledge. In view of this fact, this work had as objective the breeding of termite colonies with agricultural importance in the laboratory, of the genus Nasutitermes, to determine the necessary conditions for its development, including foraging preferences for different parts of Paspalum notatum. Young colonies were collected in field and kept in laboratory, in an artificial system with structural conditions (shelter) and environmental conditions (light, temperature, relative humidity) that sought to be the most similar to those natural ones. Different parts of $P$. notatum were offered as food option, in a test with a chance of choice. After establishing of laboratory rearing and observing the foraging of Nasutitermes spp. colonies, it was verified the preference of foraging by root, followed by decaying fragments, dry and fresh leaves, equivalently. It was also verified its adaptation to an artificial structural system composed of central box and feeding box, allowing the maintenance of the colonies for a minimum period of 5 months.
\end{abstract}

Keywords: termite breeding in laboratory; social insects; grass pests; forest pests; termites.

\section{INTRODUÇÃO}

As comunidades de cupins constituem sistemas biológicos complexos, envolvendo uma diversidade de espécies que apresentam diferentes tipos de alimentação e de nidificação. Nos ecossistemas terrestres, estes insetos são os 
maiores responsáveis pela decomposição e mineralização do carbono, influenciando as propriedades e a estrutura do solo (BIGNELL; EGGLETON, 2000). De acordo com Constantino (1999, 2002) existem cerca de 2900 espécies descritas, sendo 537 espécies encontradas na região Neotropical e aproximadamente 300 no Brasil.

Estes insetos têm chamado a atenção da comunidade científica pelo fato de serem pragas importantes em culturas diversas (BATISTAPEREIRA et al., 2004). No Brasil, muitas espécies de cupins têm sido relatadas como pragas urbanas ou pragas agrícolas, sendo presentes em todo o território nacional (CONSTANTINO, 2000, 2001; JUNQUEIRA et al., 2006; MILANO; FONTES, 2002).

Neste cenário nacional, destaca-se a espécie Heterotermes tenuis (Hagen) pelos prejuízos ocasionados em plantações de cana-deaçúcar, tendo sido relatada em canaviais dos estados de São Paulo, Mato Grosso do Sul, Minas Gerais, Goiás e Paraná (ALMEIDA et al., 2000; ARRIGONI et al., 1989), ocasionando danos que chegam à redução de 10 ton/ano de produção em campo (NOVARETTI, 1985; ARRIGONI et al., 1989). H. tenuis também já foi descrita atacando culturas de arroz, milho, algodão, soja, café, mandioca e reflorestamento de pinus e eucalipto (BERTI FILHO, 1995). Fontes e Milano (2002) também destacam a importância de espécies de Heterotermes sp como pragas de construções rurais, destruindo cercas por exemplo, além do eucalipto e cana-de-açúcar.

Assim como Heterotermes, também o gênero Nasutitermes tem expressiva distribuição geográfica no Brasil e ocorre em diferentes habitats, tendo sido encontradas espécies em pastagens, cerrados, veredas, matas (LIMA et al., 2010), alimentando-se de diferentes partes das plantas. Também é relatada como praga da cultura da cana-de-açúcar, exigindo o controle (MELO FILHO; VEIGA, 1998).

Escassos são os estudos sobre novas possibilidades de produtos e métodos de controle de cupins em área agrícola, devido ao fato de muitas espécies de importância econômica, como $H$. tenuis, serem subterrâneas e também porque não há parâmetros estabelecidos que definem um protocolo para criação de colônias de cupins em laboratório, incluindo os seus ninhos originalmente construídos no campo. Assim, há necessidade prévia de se conhecer as preferências alimentares e necessidades básicas para a sobrevivência destes insetos em meio artificial, possibilitando desta forma posteriormente desenvolver métodos visando o controle das populações em campo.

Apesar da diversidade de espécies, todos os cupins vivem em colônias (epígeas, subterrâneas, arbóreas ou no interior de construções), e se alimentam de material de origem vegetal (troncos de árvores, raízes, folhas frescas, material vegetal em diferentes estágios de decomposição), o que torna possível traçar um paralelo com relação às informações mais essenciais que permitam seu desenvolvimento fora do campo, como aspectos de microclima, sistema estrutural e alimentação.

Portanto, o objetivo deste trabalho foi estabelecer parâmetros que suportem a manutenção de colônias de Nasutitermes sp em laboratório e conhecer suas preferências alimentares por diferentes partes de $P$. notatum, permitindo assim a realização de experimentos futuros de toda natureza, incluindo aqueles relacionados ao controle das colônias, e não apenas de grupos isolados de indivíduos.

\section{MATERIAL E MÉTODOS}

As colônias de cupins usadas no experimento são provenientes da área rural do município de Indiana (SP), em área de pastagem constituída por $P$. notatum Flügge. São 3 colônias jovens pertencentes ao gênero Nasutitermes, contendo ninhos (montículos) de pequeno porte, variando entre 10 a $15 \mathrm{~kg}$, as quais, após coleta, foram mantidas no Laboratório de Entomologia Agrícola da Universidade do Oeste Paulista (UNOESTE), no Campus II, em Presidente Prudente/SP.

A coleta em campo foi realizada no mês de maio de 2016, com auxílio de 1 enxadão para possibilitar a retirada de 3 colônias inseridas ao solo, de forma cuidadosa para não quebrar nenhuma parte da estrutura dos montículos. Após serem extraídas do solo, estas foram depositadas dentro de caixas plásticas, contendo camada de $4 \mathrm{~cm}$ de gesso ao fundo, levemente umedecidas com água destilada, a fim de manter a umidade relativa do ar na parte interna da caixa. A seguir, as colônias foram imediatamente transportadas ao laboratório para início do seu estabelecimento.

Houve um período inicial de 4 meses de adaptação para os insetos, com substrato $(P$. notatum) fornecido à vontade, temperatura de 
$25-30^{\circ} \mathrm{C}$, umidade relativa do ar $60 \% \pm 5$ e ausência de luz.

As caixas plásticas utilizadas para manutenção das colônias eram fechadas, com tampa na parte superior, de cor azul escura não transparente, com dimensões de $58 \mathrm{~cm} \times 38 \mathrm{~cm}$ x $28 \mathrm{~cm}$ (comprimento $x$ largura $x$ altura). Possuía dois compartimentos anexados, em lados opostos, por um tubo plástico preto de 1 polegada de diâmetro e comprimento de $25 \mathrm{~cm}$. Tais compartimentos anexos, também de plástico não transparente, eram cilíndricos e corresponderam à caixa de alimentação $(\varnothing=35$ $\mathrm{cm}, \mathrm{h}=11 \mathrm{~cm})$ e caixa de resíduos, $(\varnothing=22 \mathrm{~cm}, \mathrm{~h}$ $=9 \mathrm{~cm})$. O substrato para forrageamento era sempre fornecido no interior da caixa de alimentação. A caixa de resíduos era mantida livre, a fim de permitir que os operários depositassem ali o possível material de descarte dos ninhos, se assim fosse o seu hábito ou eventual necessidade.

Após o período de adaptação, nas mesmas condições laboratoriais descritas, iniciaram-se os testes de forrageamento com chance de escolha, de acordo com os tratamentos da Tabela 1. A gramínea Paspalum notatum foi escolhida em função de ser a espécie predominantemente presente na área de origem das colônias, onde também se encontravam muitas colônias adultas de Nasutitermes spp. e outros cupins, plenamente estabelecidas. Para formar os tratamentos, a planta foi estratificada em diferentes partes: folhas secas, folhas frescas, fragmentos de raízes e fragmentos diversos em início de decomposição, sendo esta última composta por diferentes partes vegetais presentes na superfície da pastagem, não sendo possível saber com exatidão quais partes compunham o material.

Tabela 1. Substrato de forrageamento composto por diferentes estratos de $P$. notatum, oferecido para colônias do cupim Nasutitermes sp, em laboratório, em testes com chance de escolha.

\begin{tabular}{cc}
\hline TRATAMENTOS & Partes de $P$. notatum \\
\hline 1 & Folhas secas \\
2 & Folhas frescas \\
3 & Raízes \\
4 & Fragmentos em decomposição \\
\hline
\end{tabular}

Durante 10 dias, os substratos foram oferecidos diariamente às 3 colônias (repetições), na quantidade aproximada de $0,50 \mathrm{~g}$ por tratamento, e antes da reposição dos tratamentos eram retiradas e pesadas as sobras (massa fresca) do dia anterior. Esse procedimento foi necessário para calcular a quantia de massa fresca forrageada. Para não haver a possibilidade de fragmentos serem misturados pelos cupins, o que poderia impedir sua correta identificação, os tratamentos foram oferecidos separadamente em pequenos compartimentos, colocados simultaneamente dentro da caixa de alimentação das colônias. Esses compartimentos eram pequenos recipientes plásticos transparentes $(\varnothing=9 \mathrm{~cm}, \mathrm{~h}=$ $5 \mathrm{~cm}$ ), sem tampa, com 1 orifício lateral e próximo ao fundo, para permitir o acesso dos operários. Houve um rodízio diário na posição desses recipientes dentro da caixa de alimentação, a fim de não influenciar os resultados. Como os cupins são espécies produtoras de feromônio de trilha, cuja função é marcar quimicamente o caminho que orienta os indivíduos até a posição correta da fonte alimentar, antes do rodízio dos tratamentos para a nova posição, o fundo da caixa de alimentação também era higienizado com álcool $70 \%$. Todos estes procedimentos foram adotados para garantir que a escolha dos tratamentos pelos operários não fosse influenciada pelo resultado (posição) do dia anterior.

Os dados de consumo alimentar (massa fresca forrageada, em gramas) foram usados para calcular a porcentagem de forrageamento de cada substrato, por colônia, sendo as médias comparadas pelo teste de Tukey ao nível de 5\% de probabilidade, no software Assistat .

\section{RESULTADOS E DISCUSSÃO}

Nos primeiros 4 meses de manutenção dos cupins em laboratório, foi possível verificar 
que as colônias se desenvolveram com temperatura de 25 a $30^{\circ} \mathrm{C}$, umidade relativa do ar $60 \% \pm 5$ e ausência de luz. Em condições de microclima não favoráveis as atividades consideradas normais, isto é, rotineiras das colônias, como a coleta de substrato e a movimentação de indivíduos na caixa, começam a diminuir e podem até mesmo cessar (Ramos, comunicação pessoal). Esses relatos são inéditos e difíceis de serem comparados, pois as informações existentes até o momento na literatura sobre condições ambientais para manutenção de cupins em laboratório, são referentes a grupos de insetos isolados, fora das suas estruturas naturais (ALBUQUERQUE et al., 2008; LARANJO, 2011; SANTOS et al., 2011), mantidos em pequenos recipientes ou placas de Petri, e não no seu ninho estruturalmente intacto, em conjunto com toda colônia, como ocorreu neste experimento. Raros são os estudos com colônias fora do campo, e, quando ocorrem, as colônias ficam acondicionadas em baldes plásticos dentro de galpões, em condições ambientais não controladas (SANTOS et al., 1996) ou com pouco controle, como um sombreamento parcial de 50\% (LEITÃO-LIMA et al., 2013). Nessas situações, a manutenção das colônias é temporária, apenas por tempo suficiente para realizar os testes desejados, não havendo informações posteriores sobre o efetivo estabelecimento e adaptação das mesmas às condições descritas.

Os resultados obtidos no presente trabalho são importantes porque demonstraram a viabilidade de manter colônias de cupins em laboratório, e não apenas grupos de indivíduos, como ocorre na maioria dos ensaios produzidos até então. Albuquerque et al. (2008) relataram que o estudo de cupins em laboratório apresenta dificuldades devido à sensibilidade dos indivíduos ao serem separados da colônia, tendo como uma das consequências a redução da longevidade. Sendo assim, pode-se entender que dados obtidos em laboratório, em ensaios com colônia inteiras, são mais confiáveis, ou seja, mais compatíveis com a realidade em campo, do que aqueles provenientes de estudos conduzidos apenas com indivíduos isolados ou grupos de indivíduos retirados de seus ninhos.

Quanto ao modelo artificial para manutenção das colônias, verificou-se que a caixa plástica central, contendo o ninho, e caixa plástica anexa para alimentação foram adequadas para a criação e permitiram o desenvolvimento das mesmas, baseando-se na observação do seu intenso uso pelos indivíduos e na coleta regular do substrato fornecido como alimento. Já a caixa de resíduos não foi visitada pelos indivíduos de nenhuma das colônias deste estudo, e nenhum material de qualquer natureza foi nela depositado, sendo considerada, portanto, dispensável. Esses resultados eram esperados, já que não foram encontrados, na literatura, relatos de ninhos de cupins com câmaras para depósito de rejeitos.

Estudos dessa natureza são importantes, pois com criações de colônias efetivamente estabelecidas em laboratório, é possível desenvolver metodologias para descobrir vários aspectos ainda não totalmente elucidados sobre a ecologia de cupins, incluindo aquelas com longo tempo de condução, permitindo inclusive a realização de ensaios relacionados ao seu controle, simulando situações de campo. Os resultados produzidos terão maior confiabilidade, pois em função de não se encontrarem separados dos demais membros da colônia, mantendo as relações sociais, e nem desabrigados de seus ninhos originais, os indivíduos não sofrerão alterações de comportamento. Além disso, com uma criação laboratorial, torna-se possível executar ensaios que exigem ser replicados para as mesmas colônias em diferentes momentos no decorrer de um longo tempo, o que também aumenta a confiabilidade dos resultados.

Considerando os dados referentes à preferência alimentar, na Figura 1 é possível verificar a preferência de forrageamento de Nasutitermes sp por raizes de $P$. notatum, no entanto não houve diferença na preferência entre os substratos folhas frescas, folhas secas e fragmentos em decomposição. 
Figura 1. Forrageamento médio (\%) de colônias de Nasutitermes $s p$ em laboratório, para diferentes partes de Paspalum notatum, em testes com chance de escolha.

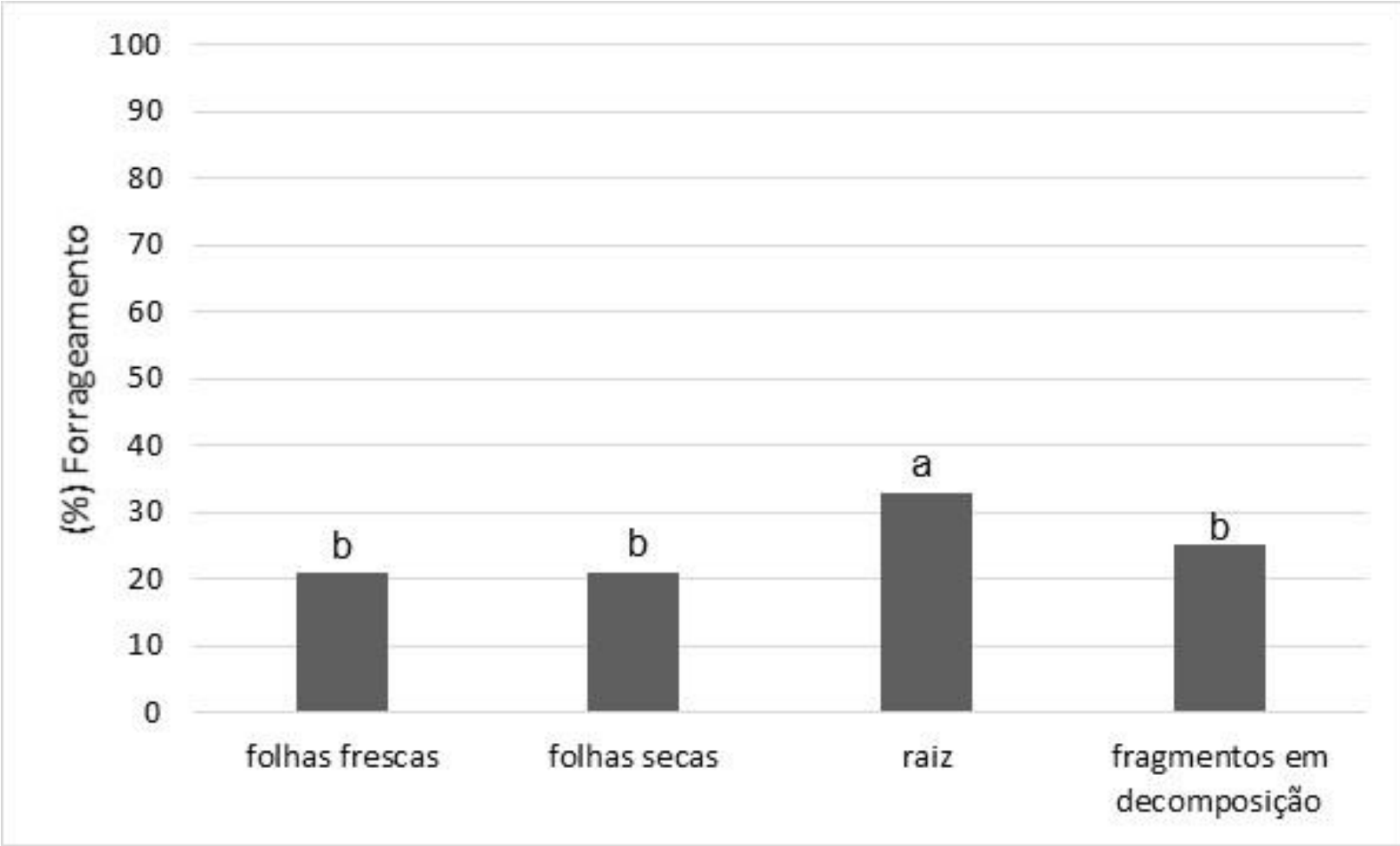

Médias seguidas de mesma letra não diferem entre si pelo teste de Tukey ao nível de $5 \%$ de probabilidade. (F-value = 4,92)

Dados sobre graus de preferência por diferentes substratos são muito importantes na condução de experimentos, para garantir que os resultados produzidos não sejam alterados em função de nutrição inadequada dos indivíduos. Laranjo (2011) descreve que a longevidade de operários de $H$. tenuis, em laboratório, foi maior quando eles foram submetidos a uma dieta de melhor qualidade. Avaliando a sobrevivência de grupos de indivíduos de Nasutitermes coxipoensis (Holmgren) em laboratório, Albuquerque et al. (2008) verificaram que o substrato cana-deaçúcar foi o que garantiu maior longevidade, quando comparado ao papel madeira picado, madeira decomposta e madeira seca. De forma semelhante, Santos et al. (2011) observaram que dietas artificiais acrescidas de nutrientes e/ou bagaço de cana também promoveram maior estimativa de sobrevivência de indivíduos de Cornitermes cumulans (Kollar) no laboratório. Em todos estes ensaios citados (ALBUQUERQUE et al., 2008; LARANJO, 2011; SANTOS et al., 2011) os indivíduos não tiveram chance de escolha quanto ao alimento oferecido, portanto não há informações quanto ao grau de preferência, ou seja, a seleção dos substratos. Escassos são os estudos que mensuram a preferência alimentar dos cupins de interesse agrícola, como aqueles conduzidos por Santos et al. (1996), verificando que, entre diversas variedades de cana de açúcar, a variedade RB72-454 foi a menos preferida pelas colônias.

Ensaios de preferência alimentar, com chance de escolha entre diferentes materiais oferecidos, também demonstram a importância da diversidade na alimentação, porque mesmo havendo um substrato preferido, ou seja, coletado em maior quantidade, os demais também são utilizados, ainda que em menores quantias. Neste ensaio verificou-se que as colônias de Nasutitermes sp, apesar de preferirem raízes, permaneceram também forrageando as folhas frescas, folhas secas e matéria orgânica (Figura 1). Fernandes e Alves (1992) também relataram que operários de $C$. cumulans preferem toletes de cana-de-açúcar, sementes de Brachiaria, sementes de milho secas ou germinadas e folhas de gramíneas secas. Estes dados indicam que na condução de experimentos laboratoriais com cupins, não é recomendado manter o fornecimento de apenas um tipo de substrato por um período prolongado, pois pode acarretar uma alteração do desenvolvimento e/ou comportamento dos indivíduos, interferindo nos resultados. A oferta inadequada de alimento pode contribuir para o declínio da colônia. 
Ao final deste ensaio, 5 meses após coleta, as colônias permaneceram vivas e em desenvolvimento, mantendo sua plena atividade de forrageamento, sem declínio aparente, simulando, portanto, uma situação muito próxima a natural. Dessa forma, utilizando-se as condições ambientais, estruturais e alimentares aqui descritas para manutenção das colônias de Nasutitermes sp, ensaios posteriores com outros objetivos poderão ser confiavelmente conduzidos, se necessário por período prolongado, simulando situações mais próximas às condições de campo.

\section{REFERÊNCIAS}

ALBUQUERQUE, A. C.; CUNHA, F. C.; OLIVEIRA, M. A. P.; VEIGA, A. F. S. L.; LIMA, E. A. L. A. Análise de substratos para testes de sobrevivência com Nasutitermes coxipoensis (Holmgren) (Isoptera: Termitidae). Arquivos do Instituto Biológico, v.75, n.4, p. 529-532, 2008.

ALMEIDA, J. E. M.; ALVES, S. B.; ALMEIDA, L. C. Controle de Heterotermes tenuis (Hagen) (Isoptera: Rhinotermitidae) e Cornitermes cumulans (Kollar) com inseticida fipronil associado ao fungo entomopatogênico Beauveria bassiana (Bals) em isca atrativa na cultura da cana-de-açúcar. Arquivos do Instituto Biológico, v.67, n.2, p. 235-241, 2000.

ARRIGONI, E. B.; ALMEIDA, L. C.; KASTEN JR., P.; PRECETTI, A. A. C. M. Distribuição de espécies de cupins, em cana-de-açúcar, em unidades cooperadas das regiões de Jaú e Sertãozinho. Boletim Técnico Copersucar, v.48, p. 38-47, 1989.

BATISTA-PEREIRA, L. G.; SANTOS, M. G.; CORREA, A. G.; FERNANDES, J. B.; ARAB, A.; COSTALEONARDO, A. M.; DIETRICH, C. R. R. C.; PEREIRA, D. A.; BUENO, O. C. Cuticular hydrocarbons of Heterotermes tenuis (Isoptera: Rhinotermitidae): analyses and electrophysiological studies. Zeitschrift fur Naturforschung, v.59, p. 135-139, 2004. https://doi.org/10.1515/znc-2004-1-226

BERTI FILHO, E. Cupins e florestas. In: BERTI FILHO, E.; FONTES, L. R. Aspestos Atuais da Biologia e Controle de Cupins. Piracicaba: Fundação de Estudos Agrários Luiz de Queiroz, 1995. p. 127-140.

BIGNELL, D. E.; EGGLETON, P. Termites in ecosystems. In: ABE, T., BIGNELL, D. E., HIGASHI,
M. Termites: Evolution, Sociality, Symbioses, Ecology. Netherlands: Kluwer Academic, 2000. p.363-387. https://doi.org/10.1007/978-94-0173223-9 17

CONSTANTINO, R. Chave ilustrada para identificação dos gêneros de cupins (Insecta: Isoptera) que ocorrem no Brasil. Papéis Avulsos de Zoologia, v.40, n.25, p. 387-448, 1999.

CONSTANTINO, R. Key to the soldiers of South American Heterotermes with a new species from Brazil (Isoptera: Rhinotermitidae). Insect Systematics \& Evolution, v.31, n.4, p. 463-472, 2000. https://doi.org/10.1163/187631200X00499

CONSTANTINO, R. The pest termites of South America: taxonomy, distribution and status. Journal of Applied Entomology, v.126, p. 355365, 2002. https://doi.org/10.1046/j.1439$\underline{0418.2002 .00670 . x}$

CONSTANTINO, R. On-line Termites Database.

2001. Disponível em:

$<$ http://www.termitologia.unb.br/index.php?opti on=com_content\&view=article\&id=10\&Itemid=1 0>. Acesso em: 25 fev. 2017.

https://doi.org/10.1163/187631200X00499

FERNANDES, P. M.; ALVES, S. B. Preferência alimentar e danos de Cornitermes cumulans (Kollar, 1932) (Isoptera: Termitidae) às plantas cultivadas em laboratório. Anais da Sociedade Entomológica do Brasil, v.21, n.2, p. 125-132. 1992.

FONTES, L. R.; MILANO, S. Termites as urban problem in South America. Sociobiology, v.40, n.1, p. 103-151, 2002.

JUNQUEIRA, L. K.; BERTI FILHO, E.; FLORÊNCIO, D. F.; DIEHL, E. Efficiency of subterranean baits for termite sampling in eucalyptus forests. Bioikos, v.20, n.1, p. 3-7. 2006.

LARANJO, L. T. Ontogenia da casta de soldados no cupim praga Heterotermes tenuis (Isoptera, Rhinotermitidae). 2011. 185f. Dissertação (Mestrado) - Universidade Estadual Paulista, Rio Claro, 2011.

LEITÃO-LIMA, P. S.; WILCKEN, C. F.; LIMA, E. V. Danos de Cornitermes cumulans Kollar, 1832 (Isoptera: Termitidae) em mudas de Eucalyptus 
grandis $\mathrm{x}$ Eucalyptus urophylla. Revista de Agricultura, v.88, n.2, p. 152-161, 2013.

LIMA, J. F. S.; CUNHA, H. F. Cupins (Isoptera) da Fazenda Serrinha na região de Miracema do Tocantins. In: SEMINÁRIO DE INICIAÇÃO CIENTÍFICA E JORNADA DE PESQUISA E PÓSGRADUAÇÃO, 8. Universidade Estadual de Goiás. Anais... Anápolis, 2010. p. 1-16.

MELO FILHO, R. M.; VEIGA, A. F. S. L. Eficiência do fipronil no controle do cupim de montículo Nasutitermes sp (Isoptera: Termitidae) em canade-açúcar. Anais da Sociedade Entomológica Brasileira, v.27, n.1, p. 149-152, 1998. https://doi.org/10.1590/S0301$\underline{80591998000100019}$

MILANO, S.; FONTES, L.R. Cupim e cidade: Implicações ecológicas e controle. São Paulo: Conquista Artes Gráficas, 2002.

NOVARETTI, W. R. T. Controle de cupins em canade-açúcar através do emprego de inseticidas de solo. Boletim Técnico Coopersucar, v.33, p. 3944, 1985.

SANTOS, A.; ZANETTI, R.; BUFALINO, L.; SILVA, W.L.P. Sobrevivência de operários do cupim de montículo Cornitermes cumulans (Kollar, 1832) (Isoptera: Termitidae) alimentados com diferentes dietas artificiais. Arquivos do Instituto Biológico, v.78, n.1, p. 151-154, 2011.

SANTOS, M. M.; LIMA, S. O.; FERNANDES, P. M. Preferência alimentar de Cornitermes cumulans e Cornitermes synderi a variedades de cana-deaçúcar em condições de laboratório. Anais da Escola de Agronomia e Veterinária, v.26, n.2, p. 65-69, 1996.

Recebido para publicação em 05/09/2017

Revisado em 01/12/2017

Aceito em 01/12/2017 